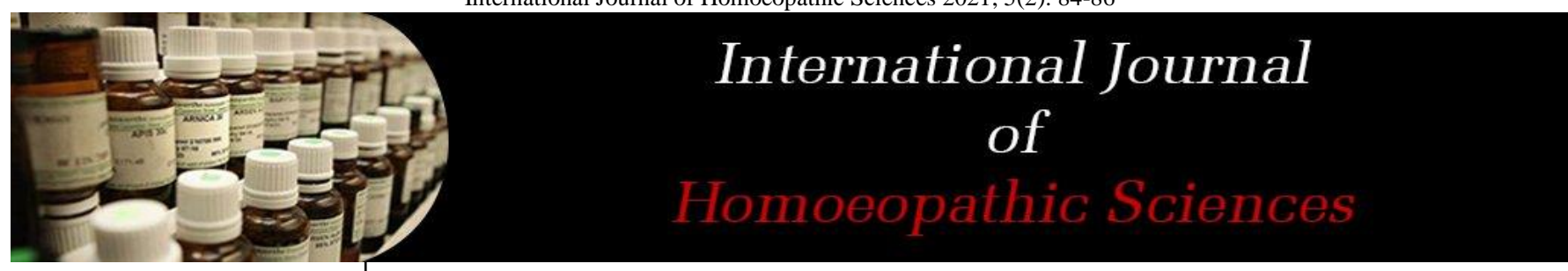

E-ISSN: 2616-4493 P-ISSN: 2616-4485 www.homoeopathicjournal.com IJHS 2021; 5(2): 84-86 Received: 08-01-2021 Accepted: 12-02-2021

Dr. Vijay Singh BHMS, MD (Hom) Scholar of Repertory, Shri Guru Nanak Dev Homoeopathic Medical College \& Hospital, Ludhiana, Punjab, India
Corresponding Author: Dr. Vijay Singh BHMS, MD (Hom) Scholar of Repertory, Shri Guru Nanak Dev Homoeopathic Medical College \& Hospital, Ludhiana, Punjab, India

\section{Renal Calculi and its Homoeopathic approach}

\section{Dr. Vijay Singh}

DOI: https://doi.org/10.33545/26164485.2021.v5.i2b.366

\section{Abstract}

Today Homeopathy is a rapidly growing system and its very popular practice in all over the world. Homoeopathy treatment is found to be more advisable because having no side effect with very safe for all ages patients Kidney stones are a major health problem today. Disordered life style, fast food habits are major reasons for health. More over medicine will prevent the future recurrence of stones. Study show that $4 \%$ of the total population has stones in urinary tract.

Keywords: Kidney stone, Renal Stone, Homoeopathic Treatment, Renal Calculi

\section{Introduction}

Nephrolithiasis (Renal Stone) is manifested by renal colic hematuria and recurrent stone formation ${ }^{[1]}$. Urolithiasis is calculus formation at any level in the urinary collection system, but most often the calculi arise in the kidney ${ }^{[3]}$. They occur frequently, as evidenced by the finding of stones in about $1 \%$ of all autopsies. Symptomatic urolithiasis is more common in males. A familial tendency towards stone formation has long been recognized ${ }^{[3]}$.

\section{Pathogenesis}

About $75 \%$ of renal stone are composed of either calcium oxalate or calcium oxalate mixed with calcium phosphate. Another $15 \%$ are composed of magnesium ammonium phosphate and $10 \%$ are either uric acid or cysteine stones. In all cases there is an organic matrix of mucoprotein that makes up about $2.5 \%$ of the stone by weight ${ }^{[1]}$.

\section{Types of stones}

\section{Oxalate stones}

- Commonest type of stone

- Also known as Mulberry stone

- Color - Brown

- Shape - Irregular

- It produced haematuria very early, disposition of blood on stone and dark color

- Hard and single, occur in infected urine

- Visualized radio logically

\section{Phosphate stones}

- Shape - Smooth, round and dirty

- Colour - White to yellow

- Commonly occurs in Renal pelvis and tend to grow in alkaline urine

- This produces recurrent urinary infectious and haematuria

- Size - Large

- It is Radio Opaque

\section{Uric acid stone}

- Shape - smooth, small, multiple, hexagonal and multifaceted

- Colour - Yellow to reddish brown

- Mostly seen in Gout, Hyperuricosuria, altered purine metabolism

\section{Cystine stones \\ - Seen in - Young Girl}


- Occurs in - Acidic urine

- Shape - Multiple, soft

- Color - Yellow and color change to greenish due to exposure

- Size - Large

- Its radio opaque because it contain Sulphuric

\section{Clinical Feature}

It's according to shape, size, position of the stone and nature. Renal calculi may be present for years without any symptom and discovered during radiological examination in different disease. This is called silent calculi.

\section{Investigation}

Plain X-ray KUB - for radio-opaque calculi. It helps to diagnosis $90 \%$ of stone. Enlarge renal shadow can also be made out

CT scan - it will identify the small missed stones. For non opaque calculi.

USG - it is the most valuable diagnosis of the stone also its location, size and confirm the enlarge kidney

Blood Examination - serum calcium, Serum uric acid, Creatinine

Urine Culture - For Protein, R.B.C, W.B.C

\section{Complication: ${ }^{[3,5]}$}

- Infection - The kidney is converted in to a bag of pus when hydronephrosis become infected and called as calculus pyonephrosis

- Anuria - Bilateral renal/ ureteric calculi. Bilateral stag horn calculi may be asymptomatic until they present with uremia

- Obstruction

- Calculus Hydronephrosis

\section{Therapeutic Aim}

To remove the stone

To prevent the complication

To prevent recurrence

\section{General Management: ${ }^{[3]}$}

More than 5 liters $/ 24$ hours fluid taken

Diet restriction according to type of stone

- If stone of oxalate-Green plantain, spinach, sweet potato, beet, currants, figs, almonds, graphs etc

- And if stone is of uric acid - Meat and meat products, shellfish, whole grain cereals. Oat meal, dried peas and bean, spinach.

\section{Homoeopathic Treatment: ${ }^{[4,5]}$}

Berberis Vulgaris: Burning pain. Pain in the bladder region. Painful left side bladder into the urethra. Blood red urine, speedily becomes turbid, deposits thick, mealy bright red sediment, slowly becoming clear but always retaining its blood.

Cantharis: Constant and intolerable urging to urinate before during and after urination. Burning, scalding urine with cutting, intolerable urging and fearful tenses or dribbing stranguary. Urine is passed drop by drop. Intolerable urging with tenses. Urine scalds the passage. Jelly like shreddy urine.
Hydrangea: Burning in the urethra and frequent desire. Urine hard to start. Heavy deposit of mucous. Sharp pain in the loins, especially left. Spasmodic stricture. Profuse deposit of white amorphous salts. Gravelly deposits.

Lycopodium: Renal colic, right sided. Pain shooting across lower abdomen from right to left. Pain in back relieved by urinating. Urine slow in Coming, must strain. Retention. Polyuria during night.

Medorrhinum: Renal colic. Painful tenesmus when urinating. Severe pain in renal region > by profuse urination. Intense pain in ureters, with sensation of passing of calculus. Urine flows very slowly. Aliments from suppressed gonorrhea. Women with chronic pelvic disorders.

Nux Vom: Renal colic is caused by a stone in the ureter, which by its irritation causes a spasmodic clutching of the circular fibers of that canal, the proper medicine relaxes these fibers and the pressure from behind forces the calculi out at once.

Ocimum Canum: High acidity, formation of spike crystals of uric acid. Turbid, thick, purelent (pyuria), bloody (hematuria), brick-dust red or yellow sediment. Odor of musk. Pain in ureters. Cramps in kidney (calculus).

Pareira Brava: Black, bloody, thick fucoid urine. Constsnt urging, great straining, pain down thighs while making efforts to maturate. Can emit urine only when he goes on his knees, pressing the head firmly against the floor. Bladder feels distended, neuralgic pain in the anterior rural region. Dribbling after micturition. Viloent pain in gains penis. Itching along the urethra, urethritis with prostatic problems. Inflammation of urethra.

Sarsaparela: Passage of gravel or small calculi, renal colic, Stone in bladder, bloody urine. Urine bright and clear but irritating. Scanty, slimy, flaky, sandy, copious, passed without sensation, deposits white sand.

Solidago: Scanty, reddish-brown, thick sediment, dysuria, gravel. Difficult and scanty. Albumen, blood and slime in urine. Pain in kidneys extends forward to the abdomen and bladder. Clear and offensive urine. Sometimes makes the use of the catheter unnecessary.

Uva Ursi: Calculus inflammation. Chronic vesicle irritation with pain, tenesmus and catarrhal discharge. Burning after the discharge of slimy urine. Frequent urging with severe spasms of the bladder. Urine contains blood, pus and much tenacious mucous with clots in large masses. Painful dysuria. Involuntary green urine. Cystitis with bloody urine.

Vesicaria: Smarting, burning sensation along urethra and bladder with frequent desire to avoid urine often with strangury. Cystitis, irritable.

\section{Conclusion}

No matter what disorders are found, every patient should be counseled to avoid dehydration and drink copious amounts of water. The efficacy of huge fluid intake was confirmed in a prospective study of first time stone formers. Increasing 
urine volume to $2.5 \mathrm{~L}$ per day resulted in a $50 \%$ reduction of stone recurrence compared with the control group [3]. Homoeopathic can be prove as beneficial to patients whom surgery is a risky afire like diabetes, hypertension etc or those search for an alternate to surgery and safe for health and for both economic or psychological reasons.

\section{Reference}

1. Kumar, Cotran, Robbins, Robbins Basic Pathology, $7^{\text {th }}$ edition, Published by Elsevier a division of reed Elsevier India Pvt. Ltd, New Delhi, 2004.

2. Anthony Fauci. Harrison's Principles of Internal Medicine $18^{\text {th }}$, Edition, Volume 2, by Anthony Fauci. Publisher: McGraw Hill Education, 2011, 2.

3. Dr. Chouhan VK. Homeopathic principles and Practice of medicine by Dr. VK Chauhan, B Jain Publishers' Ltd.

4. Boericke William. Boericke's New Manual of Homoeopathic Materia Medica with Repertory. Third revised \& Augmented Edition Based on Ninth edition, $37^{\text {th }}$ impression 2016, Published by Kuldeep Jain for B. Jain Publishers (P) LTD, 2000.

5. Dr. SK Gopi. Cure Kidney Stones with Homoeopathy First Edition, 2017. 